\title{
Lo psicologo del Ser.T. e il paziente detenuto presso la Casa Circondariale S. Vittore di Milano: fattori caratterizzanti la relazione terapeutica
}

\author{
M.A. Monti^, C. Galassi ${ }^{\circ}, B$. Ruffa*
}

SUMMARY

- The article presents a vision of the work of the Sert psychologist inside C.C. San Vittore Prison in its distinctive features compared with the assistance at a Sert territorial, such as: the type of motivation to the care of the addiction, the context Prison Institution, the passage "inside-out" and the setting.

An important factor is the "go to": in the territorial service the psychologist waits for the patient, who performs the "move to go to the service"; paradoxically, whit the detained patient is the psychologist who "go to the visiting room". A further aspect is the time of imprisonment which also affects the duration and the type of treatment.

All these factors taken together can be considered as barriers to treatment, but if well-known and shared can become important resources.

It will be through the exposure of two clinical situations that will point out the strong ambivalence that marks the difficulty in building a relationship of trust and the adaptation to a "non-traditional" setting in the psychological work $\square$.

Keywords: psychotherapy, prison, setting, addiction treatment, therapeutic relationship.

Parole chiave: psicoterapia, carcere, setting, clinica delle dipendenze, relazione terapeutica.

Il lavoro psicologico all'interno di un Ser.T. presso una Casa Circondariale è caratterizzato da alcune peculiarità, che accrescono la complessità dell'intervento e lo differenziano dall'analogo lavoro che si svolge presso Case di Reclusione e Ser.T.-Smi territoriali. Il tipo di motivazione, il contesto dell'istituzione carceraria e il passaggio "dentro-fuori" possono condizionare la relazione terapeutica, che acquisisce sfumature che andremo di seguito a tratteggiare.

Il momento dell'arresto, oltre a rappresentare una rottura con la quotidianità della propria esistenza, funziona spesso da evento precipitante che induce il detenuto a prendere atto del proprio stato e lo mette di fronte alla realtà dell'uso di sostanze; per alcuni rappresenta la prima occasione di confronto con una realtà che avevano misconosciuto, per altri la riattualizzazione di dinamiche e tematiche affrontate in altre occasioni ma non ancora risolte. Al tempo stesso la possibilità di avere accesso ad una misura alternativa al carcere per motivi terapeutici rappresenta una motivazione estrinseca, dalla quale non si può prescindere.

${ }^{\wedge}$ Psicologa Psicoterapeuta, Responsabile S.s. - C.C. San Vittore Ser.T.2, ATS Milano.

- Psicologa Psicoterapeuta, libero professionista consulente presso S.s. - C.C. San Vittore - Ser.T.2, ATS Milano e presso SMI-CAD Milano.

* Psicologa Psicoterapeuta, libera professionista consulente per S.s.C.C. San Vittore - Ser.T.2, ATS Milano.
Lo psicologo ne tiene conto quando incontra l'utente per la prima volta e avvia il processo di conoscenza, ma al contempo interroga se stesso su quanto ciò costituisca di per sé un pregiudizio nei confronti delle intenzioni del detenuto.

Il caso di A. ci offre l'opportunità di evidenziare la forte ambivalenza che contraddistingue dai primi momenti la relazione terapeutica tra lo psicologo del Ser.T. e il paziente detenuto.

A. si affaccia alla cura motivato dal vantaggio secondario che rappresenta il dichiarare la propria tossicodipendenza in carcere ed avanza delle "pretese" verso lo psicologo e l'équipe.

Riconosce la propria dipendenza ma ancora non appieno il proprio bisogno di cura e non riesce ad affidarsi agli operatori.

A. ha fatto ingresso per la prima volta in carcere presso la Casa Circondariale San Vittore di Milano all'età di 34 anni; non accetta l'imputazione per spaccio di marijuana e hashish, si sente perseguitato dalla giustizia e successivamente ritiene la condanna in primo grado troppo elevata, pertanto decide di ricorrere in appello.

Non considerando che marijuana e hashish siano delle "vere droghe", non comprende il motivo per cui si siano "accaniti" nei suoi confronti, anche a seguito di continue richieste di modifica della misura cautelare in arresti domiciliari, sempre rigettate.

All'inizio della presa in carico al Ser.T., avviata a seguito della rilevazione dello stato di dipendenza da cocaina A. si mostra poco compliante, difeso e agisce modalità manipolative e pretenziose. 
"La resistenza è la difficoltà che i pazienti provano nel rivelare a se stessi le proprie parti difficili e sofferenti e a sperimentarle nella relazione con noi. All'inizio identificano il terapeuta con i loro oggetti interni critici e per noi è molto difficile mostrare loro le parti del Sé che non approvano. Con il procedere del trattamento il paziente, utilizzando la nostra accettazione nei suoi confronti, aumenta sia in fiducia che in tolleranza verso di sé. Ciò significa che il paziente passa da un'identificazione proiettiva, nella quale il terapeuta è sentito come un oggetto antilibidi$\mathrm{CO}$, a un'identificazione introiettiva, nella quale I'oggetto antilibidico è compreso e quindi il paziente ha meno bisogno di essere severo e critico verso di sé" (Scharff, 1999).

Dai colloqui psicologici è stato possibile, nel corso della carcerazione, rilevare continui cambiamenti nelle modalità di relazione con i curanti, dovuti ai ricorrenti sbalzi d'umore e alla frustrazione data dal non vedere accolte le sue richieste da parte delle Autorità Giudiziarie: A. oscilla da modalità richiedenti e simbiotiche, a modalità svalutanti, difensive e di allontanamento, da momenti di interesse verso il proprio mondo interno a momenti in cui il pensiero e il ragionamento rimangono a un livello superficiale.

I continui cambiamenti che rileviamo in A. sono consueti in questo ambito: i pazienti, infatti, si trovano a rivedere affermazioni fatte in precedenza, ad aggiungere e smussare contenuti, così che lo stesso evento raccontato e rinarrato nel corso delle settimane si trasforma e acquisisce una nuova forma narrativa man mano che ci si lascia alle spalle la fase diagnostica e ci si avvia verso quella trattamentale.

Questo movimento trasformativo non avviene però sempre e solo in progressione evolutiva, ma registra anche battute d'arresto e involuzioni in funzione degli stati emotivi interni o di eventi esterni.

Non è infrequente, per esempio, che le vicende giudiziarie influenzino la relazione con lo psicologo del Ser.T., il quale, proprio in quanto parte integrante dell'Istituzione Carceraria, può essere vissuto come un'appendice del "sistema" che lo giudica e che gli infligge frustrazioni.

Il detenuto quando si sente "rifiutato" dalle istituzioni e dalla società reagisce spesso "rifiutando" I'aspetto trattamentale proposto, in quanto per lui incarna una contraddizione delle istituzioni stesse. Come scrive un altro detenuto sulle pagine del giornale L'Oblò del Reparto a Trattamento Avanzato "La Nave": "La privazione (...) provoca frustrazione, squilibri e rabbia che spesso si tramutano in odio, da parte non solo del detenuto ma anche della sua famiglia, nei confronti dello Stato e di tutto ciò che esso rappresenta.

L'odio diventa repulsione verso le attività rieducative messe in essere rendendo vano l'obiettivo che la pena stessa si pone come fondamento".

Lo psicologo e l'équipe hanno anche un mandato istituzionale di valutazione diagnostica ai fini dell'eventuale certificazione dello stato di dipendenza avente valore legale; può quindi accadere che, in maniera inconsapevole, il detenuto attribuisca lo stesso ruolo e lo stesso valore a istituzioni diverse ed estenda al rapporto con lo psicologo le stesse modalità di relazione e considerazione che il detenuto ha dello Stato, della Giustizia, delI'Autorità.

Talvolta accade che il paziente detenuto metta in atto meccanismi di difesa quali la scissione, distinguendo in modo netto i buoni dai cattivi: i buoni sono quelli da cui si sente ascoltato senza giudizio, da cui si sente aiutato, verso i quali nutre delle aspettative, da cui è valorizzato come soggetto e persona; i cattivi sono quelli che lo giudicano, che lo hanno rifiutato quando ha provato a riscattarsi e reinserirsi, quelli che lo umiliano facendolo sentire un oggetto, un numero, uno scarto della società. È lo stesso processo di idealizzazione e svalutazione a cui sottopone il proprio Sé e sul quale la terapia psicologica cerca di intervenire per una maggiore integrazione tra le parti.

Come vedremo, il caso di $\mathrm{A}$. è rappresentativo non solo del tipo di relazione terapeutica che si instaura "dentro" il carcere ma anche di come si può evolvere una volta "all'esterno".

A. presenta una struttura di personalità caratterizzata da tratti di immaturità e tratti narcisistici, quali senso grandioso di Sé, apparente scarsa empatia, tendenza alla manipolazione per raggiungere i propri fini, bisogno di sentirsi riconosciuto in un valore unico; si evidenzia inoltre una tendenza alla razionalizzazione, la presenza di stati di apprensione e ansia e la difficoltà a darsi dei limiti.

Durante la presa in carico al Ser.T. emerge la richiesta da parte di A. di essere agganciato a un servizio territoriale, al quale si era rivolto autonomamente per via epistolare, dove poter proseguire il programma terapeutico avviato internamente al carcere.

Dopo un anno di carcerazione viene accolta la richiesta di arresti domiciliari a casa della sorella con prescrizione di programma terapeutico ad un Servizio Multidisciplinare Integrato (SmiSer.T. privato accreditato); per garantire una continuità terapeutica, l'équipe del servizio territoriale decide di far proseguire il lavoro psicologico con la stessa psicologa che lo ha seguito durante la carcerazione e che collabora con lo Smi.

A cinque mesi dall'avvio del percorso territoriale, e a seguito della modifica della misura cautelare in una misura meno restrittiva, A. inizia a porre la questione se debba obbligatoriamente proseguire il percorso terapeutico: ritiene, infatti, di non avere più un problema con le sostanze e di non avere più bisogno di un sostegno, se non per il reinserimento lavorativo. Emerge una presa di distanza difensiva sia dal lavoro introspettivo sia dal desiderio di proseguire nell'indagare le motivazioni che lo hanno indotto a sviluppare comportamenti devianti.

Progressivamente, durante i colloqui, esprime nuovamente gli antichi atteggiamenti seduttivi e manipolatori, rendendo più complessa la possibilità di accedere al suo mondo interno.

Dopo un periodo di riflessione sull'andamento del lavoro psicologico, l'équipe decide di concludere la presa in carico psicologica a sette mesi dall'avvio del programma territoriale; a undici mesi si conclude anche la presa in cura sociale e medica dal momento che il paziente, avendo trovato un lavoro stabile e sentendosi affrancato dall'uso delle sostanze, non è più sufficientemente motivato a proseguire nel percorso terapeutico. A., seppur la terapeuta sia la stessa, non sente più la vicinanza affettiva che lo aveva agevolato nell'atto di aprirsi sulle proprie sofferenze, disattivando le proprie difese; una volta "fuori" progressivamente prende distanza dal lavoro introspettivo e riemergono rinforzate le difese a sostegno di un'organizzazione di personalità narcisistica.

Il senso del movimento di A. nel percorso di cura si comprende meglio se si considera che la relazione all'interno del carcere appare caratterizzata da un particolare clima affettivo, che difficilmente si potrà rivivere qualora la diade terapeutica si ritrovi in un servizio territoriale.

"Dentro" si condivide giocoforza una quotidianità: i colloqui vengono svolti per lo più in reparto, in piccole stanze adibite a sala colloquio, di fronte alle celle dei detenuti, mentre passa il carrello con il pranzo; i pazienti si presentano come se fossero a casa propria, in ciabatte e canottiera, offrono il caffè..

Anche i temi affrontati sono molto carichi di emotività: molte lacrime, la sofferenza dei primi giorni, la vergogna e la lonta- 
nanza dai propri affetti, l'incertezza verso il futuro e il senso di impotenza; non si tratta solo di emozioni raccontate, ma di esperienze tangibili: è l'operatore stesso che le respira mentre con pazienti percorre gli stessi corridoi.

"Fuori" la differenza di ruolo è più marcata, ognuno respira la propria aria e sceglie di quale particolare della propria vita far partecipe l'altro.

Un altro fattore che caratterizza la relazione terapeutica all'interno del carcere è "I'andare verso".

Nel servizio territoriale lo psicologo fissa un appuntamento e aspetta il paziente, che compie il movimento di "andare al servizio" così da interrogarsi ad un certo punto sul significato e sul senso di questo "andare".

Talvolta il paziente chiede "devo ancora venire?" segnalando una flessione della motivazione. È quello che succede ad A. che chiede se è ancora "obbligato" a seguire un percorso.

I pazienti detenuti, invece, non sono attivi nel movimento di andare dallo psicologo, ma aspettano di essere chiamati; eventualmente sollecitano un incontro attraverso vari canali (la domandina, il personale di polizia, i colleghi), ma sono nella condizione di attesa e impotenza.

Paradossalmente è dunque lo psicologo che compie il movimento di entrare in carcere e "andare al colloquio", peculiarità che diventa occasione per analizzare oscillazioni della propria motivazione a incontrare il paziente quali importanti segnali di controtransfert e possibili spartiacque per la ridefinizione degli obiettivi terapeutici.

Ancora una volta rileviamo l'importanza di dare ascolto al controtransfert che come sostiene Racker (1983) "rappresenta una condizione fondamentale per la ricezione delle proiezioni del paziente, che vengono organizzate in identificazioni proiettive. Questo può succedere solo senza la consapevolezza del terapeuta". Egli deve essere disponibile a ricevere queste identificazioni proiettive perché possa diventarne consapevole ed elaborare l'esperienza; solo in questo modo il paziente può sentire di essere stato profondamente compreso. Occorre anche distinguere tra controtransfert diretto, inteso come il responso al paziente, e controtransfert indiretto, inteso come risposta ad un'altra persona significante esterna al setting terapeutico (per es. istituzioni, colleghi, inviante...), presenza forte nel contesto carcerario (Schaffer, 1999).

Un aspetto fondamentale è cogliere come il detenuto risponde alla visita: c'è chi si pone in forma del tutto passiva, come se prestandosi al colloquio facesse un favore allo psicologo, vivendolo come un intervistatore da agevolare nel suo lavoro; oppure c'è chi si pone con gratitudine per questo venire incontro ai suoi bisogni di ascolto; oppure ancora chi si sorprende per la costanza con la quale lo psicologo lo chiama a colloquio.

In personalità fragili, con scarsa autostima e tratti depressivi, l'essere chiamato a colloquio può essere molto gratificante e per lo psicologo può costituire una sorta di strumento funzionale all'aggancio e all'alleanza terapeutica.

Persone con stile d'attaccamento insicuro o disorganizzato si sentono rassicurate dalla cadenza regolare e dall'affidabilità con cui lo psicologo mantiene fede agli appuntamenti dati; questo permette loro, spesso per la prima volta nella vita, di provare quello sconosciuto sentimento di fiducia verso I'Altro. II primo fondamentale pilastro su cui si baserà la relazione terapeutica. È il caso di M., che durante il primo colloquio esprime perplessità all'idea di iniziare un nuovo percorso: tossicodipendente da 30 anni è in carico da molto tempo presso un Ser.T. territoriale dal quale si aspetta solo la terapia farmacologica sostitutiva; ha subito diverse carcerazioni, in Istituti diversi, conoscendo e instaurando relazioni anche significative con molti operatori psicosociali. Ha paura e bisogno di affezionarsi al tempo stesso. $\mathrm{Al}$ secondo incontro afferma di averci pensato e di essersi accorto che il metadone non gli basta.

È al terzo incontro che si presenta con una lettera che legge con la psicologa, in cui chiede garanzie di durata sul percorso di sostegno psicologico che vorrebbe intraprendere.

È la richiesta di una promessa che non può essere siglata, ma alla quale la psicologa risponde garantendo la stessa cadenza settimanale dei primi tre colloqui e regolarizzata dalla scelta di un giorno fisso.

Ė soprattutto sul fattore temporale che lo psicologo può incidere andando a costruire un "setting": stabilendo una durata equa e costante della seduta, cadenzandola a seconda delle esigenze o delle fasi della terapia, formalizzando insieme al paziente un orario e un giorno in cui ci si incontrerà.

È sempre M. a posteriori a riconoscere questo momento come una fase di svolta nell'aggancio, che gli ha permesso di provare su se stesso fino a che punto riuscisse a fidarsi, nell'incertezza sulla durata complessiva del trattamento ma rassicurato dal sapere quale fosse il giorno deputato agli incontri di sostegno.

Quel giorno lo aiutava a scandire la settimana, a gestire I'ansia dell'attesa, a organizzare i pensieri in vista del confronto.

Già solo il fatto di sapere che sarebbe arrivato quel giorno lo faceva stare bene, quasi più dell'incontro stesso.

Il termine setting, nella sua accezione spaziale, male si adatta al contesto di cura carcerario: i rumori sono invadenti, l'odore di cibo e fumo pervade gli ambienti, i suoni e gli odori ricordano incessantemente il luogo in cui ci si trova.

Come affermato in precedenza, gli spazi della cura non vengono distinti nettamente dagli spazi del vivere in reparto: poche le sale unicamente riservate ai colloqui, spesso vengono adattate le piccole sale frigorifero, la stanza con il telefono, la sala socialità usata in compresenza con altri colleghi o con il personale volontario. Quando lo spazio di per sé non è garanzia di un significato, occorrono altre forme di tutela del setting: dalla semplice ma non scontata porta chiusa, all'allontanamento fermo dei disturbatori, per dare una forma ritualizzata al colloquio nonostante le continue interruzioni.

Anche questo è un compito dello psicologo che si mostra in qualità di garante del senso e della serietà del momento terapeutico, nonostante tutto.

"Io mi fido di lei e non so spiegarle perché ma è come se, quando ho il colloquio con lei, sono in un posto sicuro, confortevole, sereno" (M.).

Un altro aspetto importante che caratterizza il lavoro presso la Casa Circondariale, anche in confronto ad altri Istituti di Reclusione, è il tempo di permanenza che condiziona pesantemente anche la durata e la tipologia dei percorsi di cura.

Spesso il detenuto rimane presso la Casa Circondariale solo alcune settimane o pochi mesi, per poi essere trasferito - senza alcun preavviso - in altri Istituti oppure per conversione della misura cautelare in altra misura. In questo breve lasso di tempo, l'équipe curante riesce a portare a termine la prima fase di accertamento e valutazione dello stato di dipendenza e ad abbozzare un'idea di programma terapeutico.

Poco tempo a disposizione per costruire l'alleanza terapeutica necessaria ad approfondire il funzionamento della personalità e iniziare a definire gli obiettivi di cura.

Qualora, per la tipologia del paziente o per la motivazione alla cura, il tempo a disposizione risulti sufficiente all'instaurarsi di un rapporto di fiducia, spesso subentra il trasferimento ad interrompere la psicoterapia, come una frattura. 
Tale interruzione è per lo più improvvisa e priva della possibilità di chiudere adeguatamente ed affrontare le emozioni legate ai temi della separazione, della perdita o dell'abbandono, inoltre impedisce di costruire un efficace passaggio di consegne ai futuri curanti: aspetti fondamentali in qualsiasi trattamento psicologico, ma caricati di un'ulteriore valenza emotiva quando si tratta di clinica delle dipendenze proprio per le caratteristiche di personalità dei pazienti.

Non è azzardato ritenere che per alcuni individui l'uso delle sostanze rappresenti un tentativo, pur disfunzionale, di far fronte a situazioni di disagio, in particolar modo, su quella forma di depressione definita "depressione vuota" che ha origine dalla deficitaria presenza interna dell'oggetto d'amore primario (Pinamonti, Rossin, 2004).

\section{Conclusioni}

Motivazione al cambiamento e alla cura, vissuti verso l'istituzione, setting, caratteristiche emotive della relazione terapeutica e tempi di chiusura contraddistinguono ovunque il lavoro psicolo- gico, ma assumono connotazioni particolari nel contesto carcerario e, in particolare all'interno di una Casa Circondariale come San Vittore, diventano parte integrante del lavoro.

Questi fattori tendono una fune su cui si muovono gli operatori: approfondirne la conoscenza e condividerli permette di muoversi con equilibrio nel passaggio da un contesto all'altro e di trasformare in risorse potenziali ostacoli al trattamento.

\section{Bibliografia}

Caretti La Barbera (a cura di) (2005), Le dipendenze patologiche. Clinica e psicopatologia, Raffaello Cortina, Milano.

Ferrario G. (2000), "Tossicodipendenze e psicoterapia in ambito carcerario. Alcune considerazioni sul contesto", in Aa.Vv., Psicoterapia delle tossicodipendenze e dell'abuso di sostanze, FrancoAngeli, Milano.

Pinamonti H., Rossin M.R. (2004), Polidipendenze. L'assunzione multipla di sostanze in una prospettiva interdisciplinare di clinica integrata, FrancoAngeli, Milano.

Scharff D.E., Savege Scharff J. (1999), "Transfert e controtransfert", pubblicato in Interazioni, n. 2, Franco Angeli, Milano.

\section{RECENSIONE}

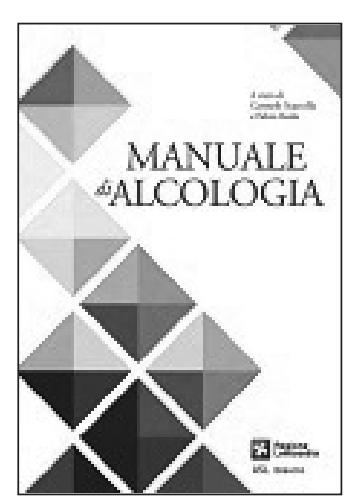

Carmelo Scarcella, Fabio Roda

\section{MANUALE DI ALCOLOGIA}

Editore: Regione Lombardia/ ASL Brescia

Gli effetti sanitari e sociali prodotti dal consumo patologico dell'alcool hanno un rilevante impatto sulla comunità.

Ciononostante non abbiamo assistito ad una decisa azione di contrasto dei fenomeni che ingenerano tale dipendenza.

Prevenzione, cura, riabilitazione sono attività non sempre efficacemente perseguite nei Servizi pubblici, soprattutto non risulta ancora vincente l'idea che solo un'azione diffusa ed integrata può consentire l'individuazione di situazioni di rischio, la precoce presa in carico e l'accompagnamento, anche nel contesto familiare, dell'assistito.

Per questo è necessario promuovere una "rete di patologia" che colleghi i servizi delle dipendenze (Ser.T. e NOA) con il sistema delle cure primarie, i presidi specialistici ospedalieri e ambulatoriali.

È indubbio che il medico di medicina generale è l'operatore sanitario potenzialmente più idoneo alla precoce identificazione di una condizione di rischio e a compiere la prima valutazione. Grazie al rapporto fiduciario che si è instaurato con l'assistito, può orientarlo verso i centri di riferimento. I medici ospedalieri avvicinando pazienti con patologie alcool correlate nel corso del ricovero possono sensibilizzarli affinché, esaurita la fase di acuzie, vengano presi in carico dai servizi per le dipendenze garantendo così una continuità tra ospedale e territorio del percorso di cura.

Il manuale di alcologia prodotto da ASL Brescia nasce dal bisogno di disporre di uno strumento di aggiornamento professionale che aiuti a formare una visione comune dell'approccio all'alcool dipendenza tra gli operatori sanitari conducendo ad un impegno efficace nell'affrontare una delle aree di fragilità più complessa.

Carmelo Scarcella, Direttore Generale dell'ASL di Brescia 